Relations industrielles

Industrial Relations

\title{
Réformes de structures et syndicalisme
}

\section{Marcel Clément}

Volume 4, numéro 3, novembre 1948

URI : https://id.erudit.org/iderudit/1023442ar

DOI : https://doi.org/10.7202/1023442ar

Aller au sommaire du numéro

Éditeur(s)

Département des relations industrielles de l'Université Laval

ISSN

0034-379X (imprimé)

1703-8138 (numérique)

Découvrir la revue

Citer cet article

Clément, M. (1948). Réformes de structures et syndicalisme. Relations

industrielles / Industrial Relations, 4(3), 21-23. https://doi.org/10.7202/1023442ar

Tous droits réservés @ C Département des relations industrielles de l’Université Laval, 1948
Ce document est protégé par la loi sur le droit d'auteur. L'utilisation des services d'Érudit (y compris la reproduction) est assujettie à sa politique d'utilisation que vous pouvez consulter en ligne.

https://apropos.erudit.org/fr/usagers/politique-dutilisation/ 


\section{Bulletin des relations industrielles}

Volume 4, numéro 3

QUÉBEC

Novembre 1948

Publié par le

Département des relations industrielles, Faculté des sciences sociales, Université Laval.

Georges-Hentu Lévesque, o.p., doyen

GÉRARD TrEmblay, directeur

GÉRARD Dion, sous-directeur

JEAN GAGNÉ, secrétaire

Charles BÉlanger, administrateur

Le Bulletin paraît mensuellement de septembre à juin (dix numéros par année). Abonnement annuel: Canada: $\$ 1.50$; étranger: $\$ 2.00$. Vingt-cinq cents le numéro.

$$
\begin{gathered}
\text { Publication répertoriée dans } \\
\text { le "Canadian Index". }
\end{gathered}
$$

Adressez toute correspondance au secrétaire de rédaction Gerard Dion

2, rue de l'Université, Québec.

Bulletin des relations industrielles

Volume 4, numéro $3 \quad$ novembre 1948

\section{Sommaire}

Réformes de structures et syndicalisme

Marcel Clément. .21

Chefs ouvriers en sessions d'études

Marcel DE LA SABLONNIÈRE. ...

Comment l'indice du coût de la vie

au ler sept. 1948 a été calculé.

Loisirs et relations de travail

Louise Dumais.

La nouvelle loi fédérale

des relations du travail

Denys Dion.

\section{Collaborateurs}

BANCroft, (Madame) M.-T., Traductrice du Bulletin.

ClÉMENT, Marcel, Licencié ès lettres, diplômé d'études supérieures de philosophie (Sorbonne), licencié en droit, diplômé d'études supérieures d'économie politique (Faculté de droit de Paris), professeur à la Faculté des sciences sociales de Laval.

de la Sablonnik̀re, Marcel, S.J., Professeur, Collège Ste-Marie, Montréal.

DumaIs, Louise, M.Sc.Soc., (Service social), secrétaire, Section d'étude des loisirs, Conseil central des oeuvres de Québec, professeur, Ecole de service social, responsable des cours sur les loisirs éducatifs à la Faculté des sciences sociales de Laval.

Dion, Denys, avocat au Barreau de Québec.

Autorisé comme envoi postal de deuxième classe, ministère des postes, Ottawa, Canada.

\section{RÉFORMES DE STRUCTURES ET SYNDICALISME}

\author{
Marcel Clément
}

Depuis que l'on voit de toutes parts le problème des réformes de l'entreprise se poser dans les esprits, il a été possible de constater deux tendances au sein des syndicats et des unions. D'une part, un grand nombre d'officiers nous l'avons constaté à la séance des ouvriers du Congrès des relations industrielles - se montrent favorables aux réformes. D'autre part, certains esprits, et non des moindres, ont marqué leur crainte de voir l'extension du mouvement des réformes de structure dans l'entreprise nuire au développement même du syndicalisme. Devant un tel état de choses, il ne suffit pas d'affirmer. Il faut examiner avec prudence. Le problème est en effet trop grave, il engage trop profondément notre avenir économique et social pour que l'on puisse se contenter d'opinions superficielles.

Le problème se pose sous deux aspects. En premier lieu sur le plan des principes. En second lieu sur le plan pratique de l'action.

\section{I}

Y a-t-il, sur le plan des principes fondamentaux qui les gouvernent, opposition ou composition, entre le syndicalisme et l'entreprise communautaire ?

Qu'est-ce que le syndicalisme? Etymologiquement, le mot, qui vient du grec, suggère une communauté d'action en vue de la justice. C'est donc là une fin éminemment positive.

Les buts les plus généraux du syndicalisme chrétien tel que l'établissent les textes pontificaux ne font que prolonger ct épanouir cette intention fondamentale de justice, - en la complétant par la charité. Dans la lettre de la Sacré-Congrégation du Concile, du 5 juin 1929, on peut lire en effet : «On doit prendre pour règle universelle et constante d'organiser et de gouverner les corporations (c'est dire, d'après le contexte, les associations syndicales) de façon qu'elles fournissent à chacun de leurs membres les moyens propres à lui 
faire atteindre, par la voie la plus aisée et la plus courte, le but qu'ils se proposent, et qui consiste dans l'accroissement le plus grand possible des biens du corps, de l'esprit, de la fortune. Mais il est évident qu'il faut viser avant tout l'objet principal qui est le perfectionnement moral et religieux. $» 1$

Il va de soi que le développement physique et culturel des travailleurs conditionné par leurs revenus économiques est le but de l'action syndicale. Elle a pour y parvenir, le choix des moyens et il est de son intérèt même de choisir la voie la plus aisée et la plus courte.

Si telle est la fin du syndicalisme chrétien, on peut se demander qu'elle est celle que poursuivent les réformes de structure au sein de l'entreprise. Sans y insister longuement rappelons que les différentes modalités reviennent toutes à appeler ouvriers et employés « à participer en quelque manière à la propriété de l'entreprise, à sa gestion, ou aux profits qu'elle apporte. ${ }^{2}{ }^{2}$ Il n'est pas douteux qu'une telle participation préconisée par l'Encyclique "Quadragesimo Anno», loin de contredire la lettre de 1929 vient au contraire deux ans plus tard indiquer un moyen sûr «d'accroître les biens du corps, de l'esprit et de la fortune.»

On peut donc affirmer, sans argumentation compliquée ni subtile, que le syndicalisme d'inspiration catholique et les réformes de structure sont deux moyens par rapport à une fin unique: le développement harmonieux de la personne humaine au sein de la société économique.

\section{II}

La question de principe réglée, on se heurte au problème pratique lui-même. Pour en comprendre toute la difficulté, il convient d'évoquer à grandes lignes ce qu'a été l'évolution syndicale, depuis son origine.

$\mathrm{Si}$, comme nous l'avons dit, le syndicalisme poursuit avant tout un fin éminemment positive, il faut bien constater cependant que c'est sous une forme négative que son action s'est développée à raison même des obstacles auxquels elle s'est heurtée. L'action concerté des ouvriers en vue de la justice sociale pouvait - idéalement - rencontrer un accueil favorable et ainsi dans un autre milieu moral que celui du libéralisme, il eut été possible de voir se développer non pas un syndicalisme de revendication mais un syndicalisme de coopération.

(1) Chevalier et Marmy, No 456, (Lettre de la S.C. à Mgr Lienart)

(2) Chevalier et Marmy, No 511, in fine, (Quadragesimo Anno).
La forme des organismes et leur but, la justice, aurait été la mème. Seule, leur attitude en face du patronat s'en serait trouvé modifiée. Au lieu du syndicalisme fermé, essentiellement dirigé pour briser une opposition, il aurait été fondé sur un exposé technique des besoins humains et sociaux et non sur une plainte humaine à forme agressive.

Historiquement, répétons-le, il n'en a pas été ainsi. C'est en violation des lois inspirées par le libéralisme, que les ouvriers ont dû tenter au début de se syndiquer, il y a de cela plus de cent ans. Aujourd'hui même, où la loi non seulement autorise, mais encore favorise le développement des organismes syndicaux et les incite clairement à passer de l'action de revendication à la collaboration patronale-ouvrière, il faut reconnaître que cette évolution se trouve entravée ou tout au moins retardée, par la méfiance réciproque qui anime le patronat devant l'action syndicale et les syndicats devant l'attitude patronale. C'est ainsi que le double syndicalisme patronal et ouvrier, et son épanouissement naturel dans l'unité: les comités paritaires, n'ont pas encore pris pleine valeur chrétienne. Nous vivons sur le plan social une douloureuse époque de transition entre le syndicalisme de revendication et le syndicalisme de coopération, entre la guerre et la paix. Si l'on tient compte de ce fait, le syndicalisme se trouve donc à la croisée de chemins. S'il cesse de revendiquer il peut craindre de se faire jouer. S'il se refuse à construire dans un esprit de coopération, il trahit sa mission de progrès social chrétien. En pratique, on peut dire que jusqu'à présent, la revendication, par la persistance de l'esprit libéral, tient nécessairement plus de place que la coopération définie comme une participation active des travailleurs à la vie économique, au terme d'une formation culturelle et sociale reçue dans leur syndicat.

Tel est le fond de scène devant lequel se joue le problème des réformes de structures. Et toutes les difficultés, qui sont réelles, viennent de là.

Comme nous l'avons déjà observé, c'est au patron qu'appartient en principe, l'initiative de telles réformes. En second lieu, leur réussite est de nature à régler de façon relativement automatique, au sein de l'entreprise, le problème, non des salaires de base, mais de leur complément social. Devant une telle perspective, est-ce que l'action syndicale, dans une telle entreprise, ne risque pas de voir son objet disparaître? Et, d'autre part, est-ce que la tentation ne sera pas bien forte, pour le patron, de retarder les augmentations légitimes du salaire de base en invoquant les participations au profit? En régime de syndica- 
lisme affaibli, l'opération se solderait par un recul douloureux.

Tel est le problème. A notre sens, le poser, c'est le résoudre. En effet, tous ces dangers n'existent que dans la mesure où leur crainte même inspirera aux officiers une attitude de passivité.

Mais cela n'est ni nécessaire, ni fatal. $\mathrm{Si}$, au contraire, le monde du travail, tout en respectant la liberté de l'initiative patronale, fait suffisamment connaître qu'il l'accueillerait avec faveur, le geste du patron se présentera comme une réponse, qui loin de nuire aux syndicats et aux unions les rejoindront dans une aspiration commune à la paix sociale. Lorsqu'il y a revendication de salaire, et transaction patronale, nous sommes en régime libéral. Mais si le monde du travail voit une aspiration qui lui est chère spontanément satisfaite par le patronat, en vue de réaliser une communauté au sein de l'entreprise, le syndicalisme est alors, en pleın, dans son rôle de progrès social, - nous sommes en régime chrétien. C'est ce qu'a admirablement exposé le président de la C.T.C.C., M. Gérard Picard, dans son discours d'ouverture du Congrès de 1948.

En second lieu, la prise en charge par les syndicats de l'étude de ces réformes aboutira normalement à faire d'eux au sein de l'entreprise, l'organisme d'exécution en collaboration avec la direction. Si la communauté de profit appelle, d̀ plus ou moins longue échéance, les comités d'entreprise, il est évident que seule la représentation syndicale peut donner à ces derniers une base suffisamment compétente et une suffisante maturité. En un tel cas, c'est la vigilance syndicale qui permettra d'éviter des confusions entre les ajustements du salaire de base, et la participation aux profits.

Ainsi, les dangers des réformes de l'entreprise ne sont sérieux que pour un syndicalisme passif ou hostile. Pour un syndicalisme progressif, elle peuvent être l'occasion providentielle de faire, entreprise par entreprise, le saut définitif qui permettra de passer de la renvendication à la collaboration, de l'opposition systématique à la composition systématique, de l'entreprise libérale à la libre entreprise. Et le caractère spontané de l'initiative patronale, sa valeur de soumission aux conseils pontificaux, sa portée en matière de collaboration patronale-ouvrière garantissent suffisamment aux patrons qui ont résolu dans leur coeur de "reconstruire l'édifice social sur l'inébranlable centre de gravité de la loi divine ${ }^{3}$ que de telles réformes sont les mesures les plus aptes à faire disparaître une méfiance ouvrière qui est finalement la mesure de leur propre méfiance. Seule, la Charité ne craint point.

(3) Pie XII, Message de Noël 1942.

\section{CHEFS OUVRIERS EN SESSIONS D'ÉTUDES}

Marcel de la Sablonnikere

Chaque fin de semaine du mois d'août dernier, on a vu arriver à l'île Saint-Ignace, des principaux centres industriels de la province, une quarantaine de chefs ouvriers, délégués officiels de leurs syndicats respectifs.

L'atmosphere. - Du vendredi soir au dimanche soir, aux conférences comme dans les discussions de groupes, dans les simples jasettes comme dans les forums du soir, c'était toujours la même atmosphère sympathique, toute de simplicité, de cette cordialité franche qui, toile de fond dans les rapports des ouvriers entre eux, en fait le charme et le bonheur.

Ce que l'on sentait chez tous, c'était ce grand désir de mieux connaître les réponses aux problèmes économiques et sociaux que posent dans leur vie de travailleurs organisés la condition actuelle de l'entreprise, ies pulsations alarmantes de la hausse du coût de la vie, avec ses pénibles répercussions sur la subsistance même de la famille ouvrière.

Le sujet de cette année. - Les chefs ouvriers avaient choisi comme sujet d'étude: le travailleur canadien devant l'inflation. « Ce n'est pas là, disait le P. Jacques Cousineau, S.J., directeur-fondateur de ces Sessions syndicales d'été, une question proprement syndicale ou, si l'on veut, elle ne touche pas à l'ouvrier en tant que producteur dans ses rapports avec des patrons. C'est le consommateur qui est ici en cause, c'est-à-dire l'ouvrier qui doit s'abriter, se nourrir, équilibrer un budget. »

Discussions en groupes. - Le cours terminé, - il y en a deux par jour - l'on se disperse par groupes de cinq ou six, à l'ombre bienfaisante des pins ou des mélèzes, sur la falaise, face au fleuve. 